\title{
Effects of THC on Behavioral Measures of Impulsivity in Humans
}

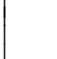

Jennifer McDonald', Laura Schleifer², Jerry B Richards ${ }^{3}$, Harriet de Wit*,I

'Department of Psychiatry, The University of Chicago, Chicago, IL, USA; ${ }^{2}$ The American Institute for Cognitive Therapy, New York, NY, USA;

${ }^{3}$ University of New York at Buffalo, Behavioral Medicine, New York, USA

This study investigated the acute effects of $\Delta^{9}$-tetrahydrocannabinol (THC) on four behavioral measures of impulsivity in recreational marijuana users. Although impulsive behavior has been studied using several different measures of impulsivity, few studies have utilized more than one of these measures on a single cohort. In this study, 37 healthy men and women participated in three sessions, in which they received capsules containing placebo, 7.5, or $15 \mathrm{mg}$ THC in randomized order under double-blind conditions. Subjects were tested on the following four tasks: the Stop task, which measures the ability to inhibit a prepotent motor response; a Go/no-go task; a Delay discounting task, which measures the value of delayed or uncertain reinforcers; and a time estimation task, which measures alterations in time perception through a time reproduction procedure. Subjects also completed mood questionnaires and general measures of performance. THC produced its expected effects on subjective measures including increases in ARCl euphoria and marijuana scales. THC increased impulsive responding on the Stop task but did not affect performance on either the Go/no-go or Delay or Probability discounting tasks. On the time reproduction task, THC increased estimates of the duration of short intervals while not affecting estimates of longer intervals. There were no significant correlations between the four tasks either before or after drug administration. These results suggest that THC may increase certain forms of impulsive behavior while not affecting other impulsive behaviors. The dissociations between the four measures of impulsivity suggest that impulsivity is an assemblage of distinct components rather than a unitary process. Neuropsychopharmacology (2003) 28, I356-1365, advance online publication, 30 April 2003; doi:10.1038/sj.npp. I 300176
\end{abstract}

Keywords: THC, cannabinoid, impulsivity, behavior, human, inhibition

\section{INTRODUCTION}

Drugs of abuse are commonly thought to induce socially unacceptable, poorly controlled, and maladaptive actions, many of which can be described as impulsive. However, few controlled studies have investigated the effects of acute doses of drugs of abuse on impulsive behavior. Moreover, there is no single, universally accepted definition or measure of impulsive behavior. We have undertaken a series of investigations into the effects of commonly abused drugs on impulsivity, using several standardized, operational measures. In the present investigation we report the acute effects of the psychoactive cannabinoid, $\Delta^{9}$-tetrahydrocannabinol (THC) on four behavioral measures of impulsivity in a cohort of recreational marijuana users.

The term impulsive is used to describe a range of maladaptive behaviors including an inability to inhibit inappropriate action, insensitivity to delayed or uncertain consequences, perception of time as progressing more

\footnotetext{
*Correspondence: Dr H de Wit, Department of Psychiatry, MC3077, The University of Chicago, 584I S. Maryland Avenue, Chicago, IL 60637, USA, Tel: + I 773702 I537, Fax: + I 7738347698 ,

E-mail: hdew@midway.uchicago.edu

Received 29 July 2002; revised 03 December 2002; accepted 27 January 2003

Online publication: 3 February 2003 at http://www.acnp.org/citations/ Npp020303308/default.pdf
}

slowly than actual time, and the perseverance of negatively reinforced actions (Ainslie, 1975; Meck, 1996; Mischel et al, 1989; Barkley, 1997; Evenden, 1999). Whether these impulsive behaviors are indicative of a monolithic phenomenon with a unitary neurobiological basis or the result of multiple, distinct processes is unclear. The few studies that have investigated the correspondence among different measures of impulsivity suggest that they may be independent (Sonuga-Barke, 2002). In addition, studies with laboratory animals indicate that different operational measures of impulsivity are mediated by different neural mechanisms (Evenden, 1999). In the present investigation we addressed this question by testing subjects with several different measures of impulsivity. The degree of correspondence between the measures both before and after drug administration may help to elucidate the underlying nature of impulsivity.

The measures of impulsivity used in this investigation are all based on different operational definitions of impulsivity and have been validated independently with populations characterized by impulsive tendencies (ie children and adults with Attention Deficit Hyperactivity Disorder (ADHD) and juveniles diagnosed with Antisocial Personality Disorder (Logan et al, 1997; Newman et al, 1985; Crean et al, 2000)). The Stop task (Logan et al, 1997) is designed to measure a subject's ability to inhibit a prepotent motoric response, with stop reaction time (SRT) as the measure of impulsivity. The Go/no-go task (Newman et al, 1985) 
involves reward/punishment conflict and the ability to inhibit a punished response. Impulsivity on the Go/no-go task is indicated by errors of commission (false alarms). The Delay discounting task (Richards et al, 1999a,b) measures the value of immediate or certain reinforcers $v s$ delayed or uncertain reinforcers. Impulsive individuals are thought to value delayed or uncertain reinforcers less than immediate or certain reinforcers (Crean et al, 2000). Finally, a time reproduction task was used to assess subjective time perception. Individuals with ADHD tend to overestimate and under-reproduce time intervals, suggestive of an increased subjective time rate (Barkley et al, 2001).

We studied the effects of THC on impulsivity for several reasons. First, marijuana is among the most commonly used psychoactive drug, being used by $76 \%$ of current illicit drug users in the United States (United States Department of Health and Human Services, 2000). While other cognitive, psychomotor, and physiological effects of acute and chronic cannabinoid use have been extensively investigated, little is known about the effects of marijuana on impulsive behavior (see de Wit et al, 1998; Ashton, 2001 for reviews of cognitive, psychomotor, and physiological effects of marijuana). Second, there is reason to believe that THC may affect impulsivity. For example, there is a long-standing, although not empirically validated, social conception that marijuana intoxication leads to maladaptive and possibly impulsive behaviors, such as crime, violence, and psychosis. A neurochemical basis for the effect of THC on impulsivity may be mediated by its indirect action on dopaminergic neurons. THC and other cannabinoid (CB1) receptor agonists increase the activity of dopaminergic neurons (Ameri, 1999). Chronic administrations of indirect dopamine agonists such as methylphenidate and amphetamine are known to decrease impulsive behavior in children with ADHD (Solanto, 1998), and acute doses of these drugs decrease impulsive behavior in laboratory studies with healthy adults (deWit et al, in press) and rats (Wade et al, 1999). This suggests that THC might decrease impulsive behavior. Marijuana also alters the perception of time, causing a pattern of estimation and reproduction similar to that observed in individuals with ADHD (Chait and Pierri, 1992; Barkley, 1997). These findings, suggesting that THC may affect impulsivity, indicate the need for systematic investigation of the effects of THC on standardized measures of impulsivity.

The primary objective of this investigation was to determine if low-to-moderate doses of THC (7.5 and $15 \mathrm{mg}$ ) influence impulsivity on four behavioral tasks: the Stop task, Go/no-go, Delay discounting, and time reproduction tasks. The second goal was to examine the correspondence of performance between the tasks. A third goal was to compare the performance of men and women. Physiological, cognitive, psychomotor, and subjective measures were obtained after placebo and THC to verify that the doses of THC used were effective.

\section{MATERIALS AND METHODS}

\section{Subjects}

Healthy men $(n=18)$ and women $(n=19)$ between 18 and 45 years of age, who reported having used marijuana at least
10 times in their lifetime, participated in this study. The study was approved by the Institutional Review Board at the University of Chicago. Subjects were recruited from the University of Chicago and surrounding community by means of posters, advertisements in newspapers, and wordof-mouth referrals. Subjects underwent a brief telephone interview to determine initial eligibility followed by a structured in-person clinical assessment. During the clinical assessment, subjects completed several diagnostic questionnaires including a psychiatric symptom checklist (SCL90; Derogatis, 1983), the Michigan Alcoholism Screening Test (MAST; Selzer, 1971), and a health questionnaire including a section on current and lifetime drug use. Subjects then underwent a psychiatric interview and physical examination including an electrocardiogram. Volunteers were excluded if they met criteria for major DSM-IV diagnoses (APA, 1994). Other criteria for exclusion included past adverse reactions to marijuana (panic or anxiety), history of drug- or alcohol-related problems (legal, family, or health problems attributable to drug or alcohol use), less than high school education, body mass index outside the range $19-26 \mathrm{~kg} / \mathrm{m}^{2}$, and smoking more than 10 tobacco cigarettes per day. Female subjects could not be pregnant or planning to become pregnant during the duration of the study, and were tested for pregnancy before each session.

Before participating in the study, subjects attended an orientation session where they provided written informed consent and were familiarized with several of the experimental procedures. The consent form stated that the study was an investigation of the effects of commonly used drugs on mood and behavior and listed several classes of drugs and possible effects that subjects might experience. For blinding purposes, participants were told they might receive a stimulant, sedative, cannabinoid, alcohol, or placebo. They were instructed to abstain from alcohol and other drugs for $24 \mathrm{~h}$ before and $6 \mathrm{~h}$ after each session, and their compliance was verified by testing breath alcohol levels (BAL) and urine samples for $\mathrm{D}$-amphetamine, cocaine, PCP, and opiates. Urine samples of females were also screened for pregnancy. Subjects were instructed not to eat for $3 \mathrm{~h}$ before the session and not to drive or operate heavy machinery for $12 \mathrm{~h}$ after each session.

\section{Design}

This study utilized a double-blind, placebo-controlled, within-subject design. Participants received a capsule containing placebo, $7.5 \mathrm{mg}$ THC, or $15 \mathrm{mg}$ THC in random order over the three test sessions, and they performed behavioral tests at the time of peak drug effect $(+100 \mathrm{~min})$.

\section{Procedure}

The study consisted of three, 4-h sessions from 3:00 to 7:00 pm in the Human Behavioral Pharmacology Laboratory (HBPL) in the Department of Psychiatry at the University of Chicago Hospitals. Sessions were a minimum of $72 \mathrm{~h}$ apart. Volunteers were tested individually in comfortably furnished rooms containing a computer for administering dependent measures, a television, and a VCR. When no 
dependent measures were being obtained, subjects were allowed to watch television, movies, and read.

Upon arrival for each session at 3:00 pm $(-30 \mathrm{~min}$ from drug administration), a urine sample was obtained for drug and pregnancy screening and BAL were checked. Baseline vital signs were also obtained at this time. At $3: 10 \mathrm{pm}$ $(-20 \mathrm{~min})$ subjects completed computerized baseline subjective effects questionnaires (described in detail below) including the Addiction Research Center Inventory (ARCI), Drug Effect Questionnaire (DEQ), and the Profile of Mood States (POMS). Baseline measures of psychomotor performance (Digit Symbol Substitution Test, DSST, Hopkins Verbal Learning Task, and Digit Span) were also completed at this time (see descriptions below). At 3:30 pm (time ' 0 ') subjects ingested a capsule containing $7.5 \mathrm{mg}$ THC, $15 \mathrm{mg}$ THC, or placebo under double-blind conditions. At 3:50 pm $(+20 \mathrm{~min})$ and $4: 50 \mathrm{pm}(+80 \mathrm{~min})$, subjects repeated the subjective effects questionnaires, DSST, and vital signs were again recorded. At 5:10 pm (+100 min) subjects completed the following four computerized behavioral tasks: Delay discounting, time test, Stop task, and the Go/no-go task (see descriptions below). At 5:50 pm (+140 min), subjects completed the subjective effects questionnaires, DSST, Hopkins Verbal Learning Task, and Digit Span. Vital signs were also recorded at this time. Subjects then completed an end-of-session questionnaire assessing their test-day experience and were transported home. After completing all three sessions, subjects attended a debriefing session where they completed personality questionnaires and were paid for their participation.

\section{Drugs}

THC (Marinol ${ }^{\circledR}, 7.5$ or $15 \mathrm{mg}$ ) was administered in opaque, colored gelatin capsules (size 00) with dextrose filler. Placebo capsules contained only dextrose. THC doses were selected because they have been shown to produce moderate behavioral and subjective effects (Kirk and de Wit, 1999).

\section{Dependent Measures}

Vital signs. Blood pressure and heart rate were measured $30 \mathrm{~min}$ before ingesting the capsule and at 20,80 , and $140 \mathrm{~min}$ after ingesting the capsule using a Digital Blood Pressure Monitor HEM-706 (Omron Healthcare, Vernon Hills, IL).

The Addiction Research Center Inventory (ARCI; Martin et al, 1971; Haertzen and Hickey, 1987; Chait et al, 1985). The ARCI is a standardized measure of drug effects. We used the 49-item version developed by Martin et al (1971), plus four additional items specific to the effects of marijuana, developed by Chait et al (1985). The resulting questionnaire consisted of 53 true/false statements describing subjective effects of various classes of abused drugs. The version of the ARCI used consists of six empirically derived scales, which measure drug-induced euphoria (morphinebenzedrine group: MBG), stimulant-like effects (amphetamine: A), intellectual efficiency and energy (benzedrine group: BG), sedation (pentobarbital-chlorpromazine alcohol group: PCAG), dysphoria and somatic effects (lysergic acid: LSD), and marijuana effects (Marijuana: M).
The Profile of Mood States (POMS; McNair et al, 1971). The POMS is comprised of 72 adjectives commonly used to described mood states. Subjects indicate how they feel at that moment in relation to each of the adjectives using a five-point scale ranging from 'not at all' (0) to 'extremely' (4). The POMS consists of 10 scales, which measure friendliness, anxiety, depression, fatigue, anger, elation, confusion, vigor, arousal, and positive mood.

The Drug Effects Questionnaire (DEQ; Johanson and Uhlenhuth, 1980). DEQ measures the subjective states 'feel drug,' 'feel high,' 'like drug,' and 'want more.' Subjects indicate on a $100 \mathrm{~mm}$ line the extent to which they feel each statement ranging from 'not at all' on the left end of the scale to 'extremely' on the right end of the scale.

The End-of-Session Questionnaire (ESQ). The ESQ asks subjects to indicate whether they liked the drug effects using a $100 \mathrm{~mm}$ line ranging from 'dislike' on the left to 'like very much' on the right. The form also requires subjects to circle which of the following statements best described the overall effects of the capsule: (1) I felt no effect at all, (2) I think I felt a mild effect, but I'm not sure, (3) I definitely felt an effect, but it was not strong, (4) I felt a strong effect, and (5) I felt a very strong effect. Subjects also indicated what they believed they had received in that session and whether they would take the drug again. Space was provided for subjects to report any unusual reactions or comments relevant to the study.

\section{General Measures of Performance}

The Digit Symbol Substitution Test (DSST; Wechsler, 1958). The DSST was used to assess psychomotor performance. The DSST is a paper and pencil task in which subjects are required to transpose a series of symbols for numbers as quickly and accurately as possible. The number of correct responses in $90 \mathrm{~s}$ was recorded. Five versions of the DSST were used to minimize learning effects.

The Hopkins Verbal Learning Test (HVLT; Brandt et al, 1992). The HVLT is a test of verbal recall consisting of immediate free recall, delayed free recall, and delayed recognition trials. A list of 12 words from four groups of semantically related words is read to the subjects, and immediately after the reading they are asked to recall as many words as possible. After $25 \mathrm{~min}$, the subjects are asked to recall the words from the list, and perform a recognition task in which they must identify words from the original list from among both semantically related and unrelated words. Six versions of the HVLT were used to minimize learning. Scores were recorded as numbers of words recalled in the free recall and delay recall trials and the number of words correctly identified in the recognition task.

Digit span (Wechsler, 1958). The Digit Span is a memory task in which subjects are read a series of numbers ranging from two to eight digits in length, and then asked to recall the series. On different trials subjects are asked to repeat the digits forward (Digit Span Forward) or backward (Digit Span Backward). Six versions of the Digit Span were used to reduce learning across trials. 


\section{Behavioral Measures of Impulsivity}

The Stop task (Logan et al, 1997). The Stop task is designed to assess a subject's ability to inhibit a prepotent motoric response. Subjects are instructed to respond as quickly as possible when a certain letter (go signal) appears on a computer screen, and to inhibit their responses when a tone is heard (stop signal). The tone is presented on random trials and at different delays following the letter presentation on $25 \%$ of the trials. The delays to the stop signal are varied systematically according to the subject's performance: the delay to the tone is adjusted until the subject inhibits his or her responses on approximately $50 \%$ of trials. After the stop signal delay has been adjusted to this $50 \%$ criterion, the time required for the subject to inhibit his/her go response can be determined. The SRT is calculated by subtracting the final mean delay at which the tone is presented from the mean go reaction time (GRT). Both GRT and SRT are measured in milliseconds

The Go/no-go task (Newman et al, 1985). The Go/no-go task is a learning task designed to assess the ability to inhibit an inappropriate response. The task consists of repeated presentations of eight numbers, of which four are designated 'correct' and four 'incorrect'. The 'correct' and 'incorrect' numbers are changed for each of the three test sessions, so that the subject is required to learn the correct and incorrect numbers during the test session. The subject's task is to respond to the correct numbers, and to withhold responses to the incorrect numbers. Subjects receive rewards (10 cents) for correct responses and are penalized for incorrect responses ( -10 cents). The outcome measures are errors of omission (withholding a response when a 'correct' stimulus is presented) and errors of commission (ie responding to an 'incorrect' stimulus). Subjects received the amount of money earned at the end of the session.

Time test (University of Massachusetts Medical Center). The time test assesses the subject's ability to reproduce varying intervals of time. Two light bulbs appear on the computer screen and one is illuminated for a time interval of $2,4,8,16$, or $32 \mathrm{~s}$. Immediately after presentation, the subject is asked to use the spacebar to illuminate a second light bulb for the same amount of time. Subjects are instructed not to count, and to minimize this tendency, they are instructed to count the number of small figures that appeared at irregular intervals on the screen.

The Delay discounting task (Richards et al, 1999a,b). Delay discounting provides an index of the relative value of immediate $v s$ delayed rewards. This task uses a computerized adjusting amount procedure to measure discounting of delayed and uncertain reinforcers. Subjects have the opportunity to choose between different amounts of money available after different delays or with different probabilities. The test consists of approximately 100 questions, such as: (1) Would you rather have $\$ 10.00$ in 30 days or $\$ 2.00$ at the end of the session, or (2) Would you rather have $\$ 5.00$ for sure or $\$ 10.00$ with a $25 \%$ chance? The questions are presented on a computer screen according to an adjusting amount procedure (Richards et al, 1999a, b), in which the amount of immediate, certain money is adjusted across successive questions (trials) until an amount is reached that is judged by the participant to be equivalent to the delayed (delay trials) or uncertain $\$ 10$ reward (probability trials). The amount of immediate, certain money the subject judges to be equivalent to the $\$ 10$ reward provides the indifference point, a quantitative measure of the subjective value of the delayed and uncertain rewards. Delay and Probability trials are intermixed. Within each session, indifference points are determined for five different delays $(0,2,30,180$, and 365 days) and five different probabilities $(1.0,0.9,0.75,0.5$, and $0.25)$. On probability trials, participants choose between a variable amount of money delivered for sure (probability $=1.0$ ) and $\$ 10$ to be delivered on a probabilistic basis. The indifference points obtained at each of the delays and probabilities are then plotted to form two discount functions. The delay discounting function is derived through curve-fitting analyses. The delay indifference points were fit to the equation

$$
\text { Value }=A /(1+k D),
$$

where $A$ is the amount of the reward, $D$ is the delay to reward, and $k$ is a free parameter. Larger values of $k$ indicate more marked devaluation of reinforcer value by delay, and thus greater impulsivity. The probability indifference points were fit to the equation

$$
\begin{aligned}
& \text { Value }=A /(1+h O), \\
& O=(1 / p)-1,
\end{aligned}
$$

where $p$ is the probability of reward and $O$ indicates odds against. In this formulation, the value of $h$ indicates how rapidly the value of a reward decreases as the probability of its occurrence decreases. Higher values of $k$ and $h$ indicate greater impulsivity (greater discounting of delayed or probabilistic $v s$ certain immediate rewards). Equations (1) and (2) were fit to the five delay indifference points using a nonlinear curve-fitting program (Origin 4.1, 1995). This curve-fitting program determined the best-fitting values for $k$ and $h$ and the coefficient of determination for delay and probability discounting. In plotting the discount functions for probability, we have chosen to plot the data with 'odds against winning' (rather than probability) on the $x$-axis in order to make clear the hyperbolic nature of the discount function. Odds against winning for a given probability value is provided by computing $O$ in Equation (3); thus the five probability values used in this study $(1.0,0.9,0.75,0.5$, and 0.25 ) were converted to $0,0.11,0.33,1.0$, and 3 . Previous research has shown that the hyperbolic discount equations used in this analysis describe the discounting better than alternative equations such as an exponential. For a detailed discussion of the rationale for using the hyperbolic discount equation and the curve-fitting procedure, see Richards et al (1999a, b).

\section{Personality Questionnaires}

The Barratt Impulsiveness Scale-11 (BIS-11; Patton et al, 1995). The BIS-11 was used to assess impulsivity as a personality trait. The questionnaire consists of 30 statements to which subjects respond by choosing one of the following responses: rarely/never, occasionally, often, and almost always. 


\section{Data Analysis}

Data analyses were conducted using Statistica ${ }^{\circledR}$. Two-way, repeated measures analyses of variance (ANOVA) were used to examine the effects of drug (three levels: placebo, 7.5, and $15 \mathrm{mg}$ ) over time (four levels: -20 (baseline), 20, 80, and $140 \mathrm{~min}$ ) for vital signs, subjective measures, and general measures of performance. Data from the behavioral measures of impulsivity were analyzed using one-way ANOVAs (three levels: placebo, 7.5, and $15 \mathrm{mg}$ ). Post hoc comparisons were conducted using the Tukey honest significant difference test. Sex differences were examined by including a between-subject factor in the ANOVA. Pearson product-moment correlations were used to assess intertask performance correlation both before and after drug. The significance level for all statistical tests was $p<0.05$.

\section{RESULTS}

\section{Subject Demographics}

Subject demographics and drug use histories are summarized in Table 1. Subjects ranged in age from 18 to 36, with the majority of subjects younger than 25. A total of $68 \%$ were full-time students at the time of the study.

\section{Vital Signs}

THC dose-dependently increased heart rate, compared to placebo (Figure 1). Both the 7.5 and $15 \mathrm{mg}$ doses increased heart rate after ingestion of the capsule, with the high dose increasing heart rate more than the low dose. The drug effect peaked at $80 \mathrm{~min}$ for both doses. THC did not significantly affect systolic or diastolic blood pressure.

\section{Subjective Effects}

THC significantly increased subject ratings of stimulant-like effects, marijuana-like effects, dysphoria, euphoria and somatic effects, and sedation on the ARCI A, M, LSD, $\mathrm{MBG}$, and PCAG scales respectively, and decreased ratings of intellectual efficiency and energy on the BG scale. Tukey HSD post hoc analysis revealed that the $7.5 \mathrm{mg}$ dose
Table I Subject Demographics and Drug Use Summaries $(N=37)$

\begin{tabular}{|c|c|}
\hline Age (mean, SD years) & $23 \pm 4.48$ \\
\hline Weight (mean, SD lbs) & $144.62 \pm 23.83$ \\
\hline Sex (male/female) & $19 / 18$ \\
\hline Race (Cauc/Black/Asian) & $27 / 4 / 6$ \\
\hline \multicolumn{2}{|l|}{ Education (n) } \\
\hline High school/partial college & $1 / 16$ \\
\hline College degree/adv degree & $7 / 13$ \\
\hline Full-time student (n) & 25 \\
\hline \multicolumn{2}{|l|}{ Current drug use (mean $\pm S D$ ) } \\
\hline Alcohol (drinks/week) & $5.97 \pm 5.22$ \\
\hline Caffeine (drinks/week) & $11.14 \pm 10.81$ \\
\hline Cigarettes (cigarettes/week) & $8.88 \pm 17.53$ \\
\hline Marijuana (times/week) & $1.55 \pm 2.02$ \\
\hline \multicolumn{2}{|l|}{ Lifetime drug use } \\
\hline Stimulants ( $n$; ever used) & 12 \\
\hline Tranquilizers ( $n$; ever used) & 6 \\
\hline Hallucinogens ( $n$; ever used) & 25 \\
\hline Opiates ( $n$; ever used) & 6 \\
\hline \multicolumn{2}{|l|}{ Marijuana } \\
\hline Used 10-20 times $(n)$ & 7 \\
\hline Used 20-100 times (n) & 13 \\
\hline Used $>100$ times $(n)$ & 17 \\
\hline Inhalants ( $n$; ever used) & 13 \\
\hline
\end{tabular}

increased scores on the M, LSD, and PCAG scales, relative to placebo, after 80 and $140 \mathrm{~min}$. Figure 1 shows the time course of effects on the ARCI M scale and the timing of the behavioral tasks. The $15 \mathrm{mg}$ dose significantly increased scores on these scales relative to both placebo and the $7.5 \mathrm{mg}$ dose, at 80 and $140 \mathrm{~min}$. Both doses significantly increased scores on the A and MBG scales, relative to placebo, at 80 and $140 \mathrm{~min}$.

THC dose-dependently increased ratings on the DEQ 'feel drug', 'feel high', and 'want more' scales. Both doses significantly increased scores on 'feel drug' and 'feel high' scales at 80 and $140 \mathrm{~min}$, while they increased ratings on the 'want more' scale only at $140 \mathrm{~min}$.

THC also dose-dependently and significantly increased scores on the POMS anxiety, fatigue, anger, and confusion
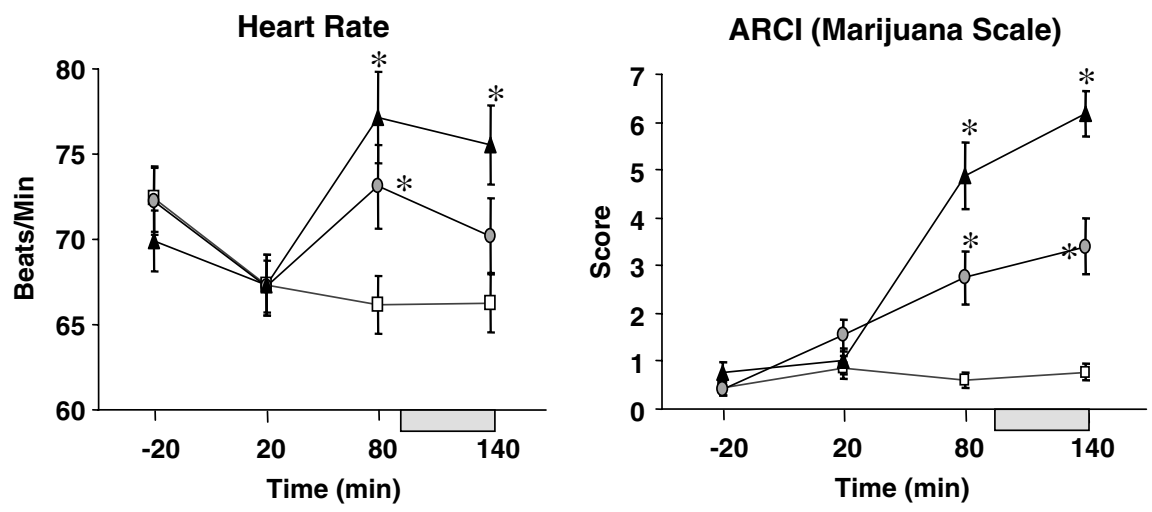

Figure I Mean ( \pm SEM) heart rate and scores for ARCI M scale for placebo (squares), $7.5 \mathrm{mg}$ THC (circles), and I $5 \mathrm{mg}$ THC (triangles) sessions, at baseline (-20) and 20,80, and 140 min after capsule. The shaded region along the $x$-axis shows when impulsivity tasks were performed, near the time of peak drug effect. Asterisks indicate significant difference from placebo. 
Stop Reaction Time

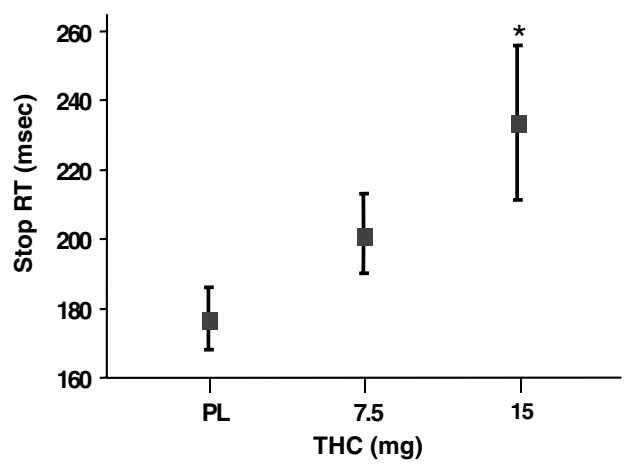

Go Reaction Time

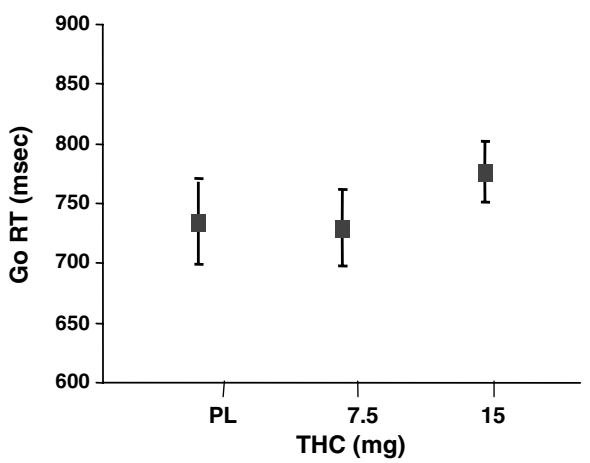

Figure 2 Mean Stop task SRT and GRT ( \pm SEM) for placebo, $7.5 \mathrm{mg}$ THC, and $15 \mathrm{mg}$ THC. The asterisk indicates significant difference from placebo. THC increased SRT without increasing GRT, suggesting the drug specifically altered response inhibition.

scales. The POMS arousal scale was significantly lower than placebo at $140 \mathrm{~min}$ for both the 7.5 and $15 \mathrm{mg}$ doses.

\section{General Measures of Performance}

THC did not significantly affect scores on the DSST, Hopkins Verbal Learning Task, or the Digit Span Forward. However, both doses of THC significantly impaired performance on the Digit Span Backward at $140 \mathrm{~min}$.

\section{Behavioral Measures of Impulsivity}

On the Stop task, the $15 \mathrm{mg}$ dose of THC significantly increased SRT on the Stop task $\mathrm{F}(2,70)=5.07 ; p<0.01$ (Figure 2). THC did not affect GRT, suggesting that the observed effect was specific to response inhibition (Figure 2).

THC did not affect performance on the Go/no-go task (Figure 3). The drug did not affect either errors of omission or errors of commission, and it did not affect the reaction time on correct responses.

THC significantly impaired performance on the time reproduction task $\mathrm{F}(8,288)=2.51 ; p<0.05$ (Figure 4). The $15 \mathrm{mg}$ dose significantly increased reproductions at the 2 and $4 \mathrm{~s}$ intervals.

THC did not significantly affect either delay or probability discounting. For reasons that are not known (see Discussion), about half of the subjects in this study did not discount the delayed values to any appreciable amount, yielding negative $R^{2}$ and making it impossible to calculate the $k$ values. Therefore, ANOVAs of the $k$ values for delay were conducting using the 16 subjects whose $R^{2}$ values were positive. The mean and standard deviation $\log k$ value for these subjects in the placebo, 7.5, and $15 \mathrm{mg}$ conditions were $-1.84(0.61),-1.87(0.71)$, and $-1.68(0.70)$, respectively (NS). The corresponding mean $R^{2}$ values were $0.88,0.84$, and 0.83 . Figure 5 (left) shows the median indifference points from all 36 subjects, and the hyperbolic function that fits these points. On the probability discounting task, all subjects had positive $R^{2}$ values. The mean and standard deviation $\mathrm{h}$ values in the placebo, 7.5 , and $15 \mathrm{mg}$ conditions were 1.79 $(0.50), 1.74(0.49)$, and $2.0(0.48 ; \mathrm{NS})$, and the corresponding $R^{2}$ values were $0.87,0.85$, and 0.90 . Figure 5 (right) shows the median indifference points for the 36 subjects, and the hyperbolic function that best fit these points.

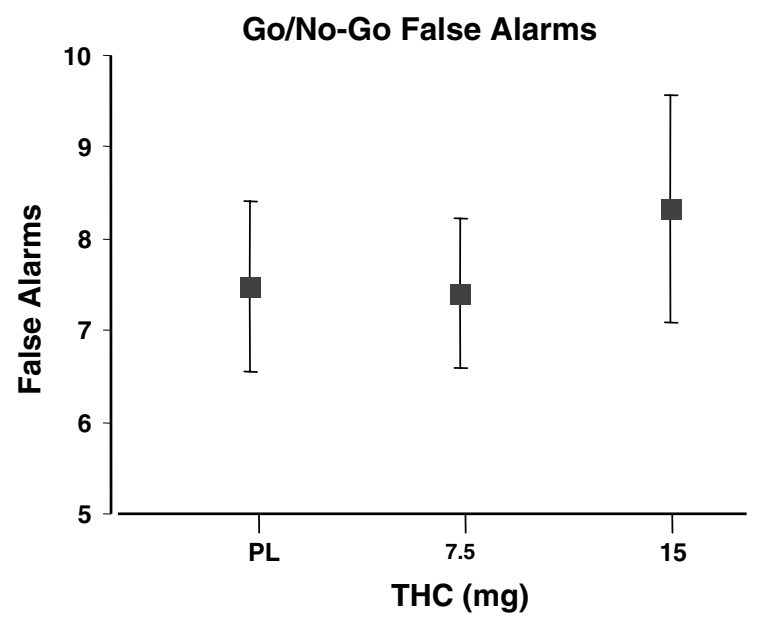

Figure 3 Mean $( \pm$ SEM) number of false alarms for the Go/no-go task. THC did not affect performance on this task.

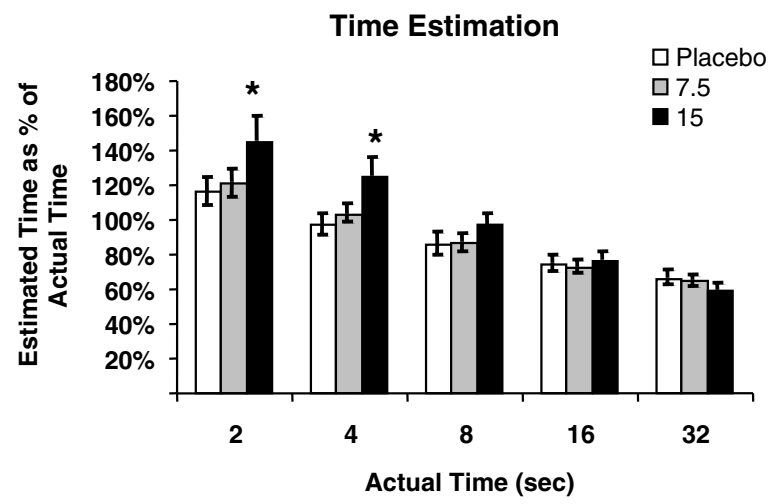

Figure 4 Reproduced time presented as mean percentage of actual time ( \pm SEM) for placebo, $7.5 \mathrm{mg}$ THC, and $15 \mathrm{mg}$ THC. Asterisks indicate significant difference from placebo within the time interval.

\section{Gender Differences and Intertask Correlation}

No significant correlations were found among the tasks either before or after drug administration, with $r$ values ranging from -0.287 to 0.320 . We examined sex differences in impulsivity by comparing scores on the behavioral measures of impulsivity between males and females on 


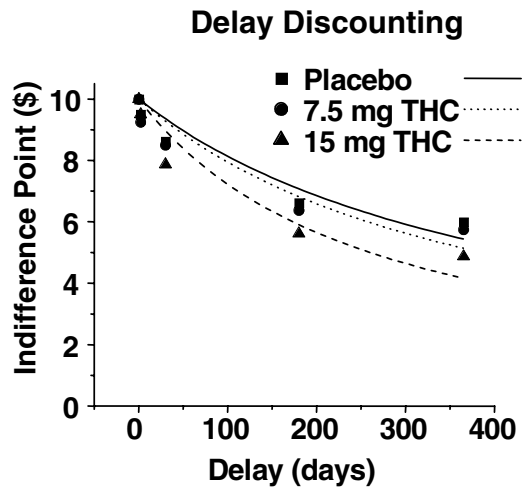

Probability Discounting

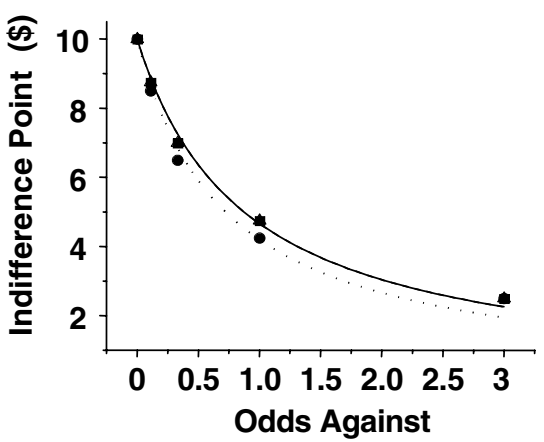

Figure 5 Median indifference points and hyperbolic discount functions for delay (left panel) and probability (right panel) discounting after placebo, $7.5 \mathrm{mg}$ THC, and $15 \mathrm{mg}$ THC. THC did not significantly affect either delay or probability discounting.

placebo sessions and also in response to THC. There were no significant sex differences in performance on the impulsivity measures.

\section{Personality Data}

The mean score on the Barratt Impulsiveness Scale for the whole cohort was $64 \pm 10.7$. This value is the same as the mean value reported by Barratt in the development of the scale, using a comparable population (Patton et al, 1995). Although we were initially interested in the effects of THC on the tasks in relation to trait levels of impulsivity, there was not enough variability in the BIS scores to make this analysis meaningful.

\section{DISCUSSION}

The primary goal of this study was to determine how THC affects performance on four behavioral measures of impulsivity. The main findings from this study were that THC increased impulsive responding on the Stop task and decreased patterns of impulsive responding on the time reproduction task. THC did not significantly affect either the Go/no-go or Delay discounting tasks. There were no significant correlations between the four impulsivity tasks across individuals either before or after drug administration. Together, these findings suggest that multiple processes underlie impulsive behavior, and that THC affects some, but not all, of these processes.

THC significantly increased the amount of time necessary for subjects to inhibit a prepotent response on the Stop task, while having no effect on GRT. This indicates that the effect of THC was specific to response inhibition and not an artifact of slowed reaction time. Previous studies indicate that the Stop task is sensitive to the type of drug administered and that different classes of drugs produced markedly different effects. For example, we have found that ethanol produces effects similar to those observed here with THC (de Wit et al, 2000). Moderate doses of ethanol (0.4 and $0.8 \mathrm{~g} / \mathrm{kg}$ ) increased SRT without slowing GRT. In contrast, $20 \mathrm{mg}$ of D-amphetamine decreased SRT (de Wit et al, 2002). Notably the two drugs with more sedative-like effects (THC and alcohol) increased impulsive responding, whereas the stimulant drug (D-amphetamine) decreased impulsive responding. This suggests that the effects of THC and alcohol may be related to overall alertness; however, several observations do not support this interpretation. First, GRT is a control condition to detect nonspecific sedation, and neither of these drugs decreased GRT. Second, in the present study THC did not significantly impair performance on the DSST, a measure of nonspecific motor performance. These findings suggest that the effect of THC on SRT is independent of the drug's effects on overall alertness.

THC did not have an effect on Go/no-go performance. Although this finding is apparently inconsistent with findings from the Stop task, the Go/no-go task differs from the Stop task in important ways. In the Go/no-go task the go (rewarded responses) and stop signals (punished responses) are presented independently and subjects must choose whether or not to respond to each signal. In contrast, in the Stop task the stop signal (tone) occurs simultaneously with the go signal (visual cue). The Go/no-go paradigm puts a greater load on cognitive inhibition, involving response selection, while the Stop task requires the retraction of a response already initiated by the go signal, or motor inhibition (Rubia et al, 2001). These components of inhibitory control may be independent. The lack of effect of THC on a task that requires cognitive inhibition is consistent with findings of a recent study in which acute marijuana intoxication did not affect accuracy on measures of cognitive functioning (Hart et al, 2001). Thus, it is possible that THC may cause deficits in motor inhibition that affect performance on the Stop task, while not significantly impairing inhibitory processes that are more cognitively driven.

An alternative explanation for the lack of effect of THC on the Go/no-go task is that this specific task may not be sensitive to variations related to mild drug effects or subtle intersubject variations. This task has not been tested extensively with healthy populations or with acute drug administration; therefore, its sensitivity is not known. However, we recently conducted a study in which Damphetamine decreased the number of errors of commission, indicating that this task is at least sensitive to improvement by drugs (de Wit et al, 2002). Further investigations of the effects of drugs or other manipulations using this task are needed before the present findings with THC can be fully interpreted. 
Previous research has demonstrated that marijuana alters time perception (Chait and Pierri, 1992; Schulze et al, 1988). Marijuana causes the overestimation and under-reproduction of time intervals, suggesting a speeding of the internal clock. Contrary to these findings, in the present study, the $15 \mathrm{mg}$ dose of THC caused subjects to significantly overreproduce the 2- and 4-s time intervals on the TimeTest. While the reason for these conflicting results is not clear, one explanation is that the intervals used in this task were relatively short. In earlier research the reproduction intervals tested have typically been $10 \mathrm{~s}$ or longer and the reported effect of under-reproduction has rarely occurred at intervals less than $30 \mathrm{~s}$ in length (Chait and Pierri, 1992). Although no significant drug effects were observed in this study at the longer time intervals $(8,16$, and $32 \mathrm{~s})$, there was a nonsignificant trend toward under-reproduction at the longest time interval (32 s). The observed effect at the 2- and 4 -s intervals may be due to other nonspecific performance effects of THC or its effects on motor inhibition. The task requires subjects to reproduce time intervals by holding down the spacebar on a keyboard. Just as THC reduced the ability to inhibit an ongoing response on the Stop task, THC may have interfered with the ability to discontinue holding down the spacebar, resulting in significantly longer time reproductions at the shortest intervals.

THC had no observable effect on the value of delayed or uncertain rewards as measured by the Delay discounting task. As in the case of the Go/no-go task, the sensitivity of this task to acute drug effects is uncertain. The Delay discounting procedure has been used extensively in humans to characterize stable individual differences in discounting (Crean et al, 2000; Madden et al, 1997; Petry, 1999; Green et al, 1994; Mitchell, 1999; Rogers et al, 1999). For example, drug users, gamblers, psychiatric patients exhibiting symptoms of impulsivity, and patients with specific cortical lesions all exhibit more pronounced reward discounting than appropriate comparison groups. However, there are a few reports showing momentary changes in delay discounting, for example after treatment with a drug or after an acute experimental treatment. Although we found a small decrease in discounting after $15 \mathrm{mg} \mathrm{D}$-amphetamine (de Wit et al, 2002), two other treatments that might be expected to increase impulsive behavior, alcohol and decreased brain levels of serotonin by tryptophan depletion, had no effect (Richards et al, 1999a,b; Crean et al, 2002). This is in contrast to studies with laboratory animals, in which several studies have reported acute changes in discounting after administration of drugs (Evenden and Ryan, 1999; Richards, 1999; Wade et al, 2000). There are several reasons why the delay discounting procedure may be relatively insensitive to state changes in impulsive behavior in humans. For example, the time frames involved in the human delay discounting procedure range from days to months. Thus, subjects are required to make decisions about rewards that will be obtained well after the drug's effects have dissipated. The fact that their decisions during the procedure will have consequences well after the drug is gone may affect subjects' decisions. In contrast, in the studies with laboratory animals, both the decisions and the consequences of the decisions occur while the animal is in the drugged state. Another factor that might affect whether the procedure is sensitive to state changes in impulsivity is the hypothetical nature of the rewards. In this study the subjects did not actually receive the monetary rewards resulting from their choices, and the hypothetical outcomes were sometimes in the distant future (ie months later). Thus, the outcomes are not actually experienced during the sessions, in the 'drugged' state. Although it was recently shown that individual differences in delay discounting were the same regardless of whether rewards were real or hypothetical (Johnson and Bickel, 2002), it is not known if this also applies to state changes in impulsive behavior. Indeed, compared to previous studies conducted in our laboratory in which monetary rewards were actually given after the sessions (Richards et al, 1999; de Wit et al, 2002), the degree of delay discounting in this study was low: many of the subjects valued rewards delayed by as long as a year in the future only slightly less than immediate rewards. Thus, it is possible that more pronounced delay discounting, and more pronounced drug effects, would have been obtained if the rewards had been real rather than hypothetical. Another factor that may have affected the sensitivity of the Delay discounting task in the present study is that the value of delayed reward was rather low (\$10.00) and it is possible that drug-induced changes in impulsive behavior would be observed with higher stakes. Further studies are needed to determine whether the delay discounting procedure can be modified to increase its sensitivity to state changes in impulsive behavior.

Another goal of this study was to determine if performance on the different tasks was correlated, both in the absence of the drug (at baseline) and in response to the drug. As discussed earlier, impulsivity is a poorly defined construct that is used to describe a range of behaviors. The tasks used in the present investigation all measure behaviors that are described as impulsive, but it is unclear whether they result from a single underlying process or are distinct components of impulsivity. The lack of correlation between the measures of impulsivity, both on the placebo session and in response to THC, suggests that separate processes are involved. The current findings are consistent with two other studies conducted in our laboratory using the same four tasks (de Wit et al, 2002, unpublished data). Moreover, a recent investigation that tested a cohort of ADHD children on both a measure of delay aversion and a task similar to the Stop task concluded that delay aversion and poor inhibitory control are central, but unrelated, characteristics of ADHD, a disorder defined by impulsive behaviors (Sonuga-Barke, 2002). Where exactly the lines are to be drawn between the different components or varieties of impulsivity is beyond the scope of this paper. Further studies in which individuals are tested on several measures of impulsivity will help to identify and characterize these different components. Additionally, the effects of pharmacological intervention in both human and animal models can aid in elucidating the neurobiological basis of the various processes that underlie impulsive behaviors.

The present research is limited by several factors. As already emphasized, it is difficult to interpret THC's lack of effect on several of the tasks because the tasks have not been extensively used for measuring acutely induced alterations in impulsivity. This may have been further confounded by the fact that our subject sample was low on the trait of impulsivity as measured by standardized personality 
questionnaires. It is possible that the drug may produce greater effects on impulsive behaviors in individuals who are high on the trait of impulsivity, as measured by personality questionnaires.

In conclusion, THC was found to increase impulsive responding on the Stop task while not meaningfully affecting performance on the other three measures of impulsive behavior. These findings emphasize the importance of utilizing multiple measures when investigating impulsive behavior. To say that drugs of abuse, such as marijuana, affect impulsivity, when impulsivity is taken as a nonspecific construct, may not give an accurate or meaningful description of the drug's effects. The present findings suggest that it is possible for drugs to affect some processes that may lead to impulsive behaviors while not affecting others. Controlled studies utilizing pharmacological manipulations of known action in the brain will aid in discovering the neurobiological basis of impulsive behaviors and how they are interrelated.

\section{REFERENCES}

Ainslie G (1975). Specious reward: a behavioral theory of impulsiveness and impulse control. Psychol Bull 82: 463-496.

Ameri A (1999). The effects of cannabinoids on the brain. Prog Neurobiol 58: 315-348.

American Psychiatric Association (1994). Diagnostic and Statistical Manual of Mental DISORDERS, DSM-IV, 4th edn. American Psychiatric Association: Washington, DC.

Ashton CH (2001). Pharmacology and effects of cannabis: a brief review. Br J Psychiatry 178: 101-106.

Barkley RA (1997). ADHD and the Nature of Self-control. The Guileford Press: New York.

Barkley RA (2002). TimeTest application. University of Massachussets Medical Center, Massachussets.

Barkley RA, Murphy KR, Bush T (2001). Time perception and reproduction in young adults with attention deficit hyperactivity disorder. Neuropsychology 15: 351-360.

Brandt J, Corwin J, Krafft L (1992). Is verbal recognition memory really different in Huntington's and Alzheimer's disease. J Clin Exp Neuropsychol 14: 73-84.

Chait LD, Fischman MW, Schuster CR (1985). 'Hangover' effects the morning after marijuana smoking. Drug Alcohol Depend 15: 229-238.

Chait LD, Pierri J (1992). Effects of smoked marijuana on human performance: a critical review. In: Murphy L, Barke A (eds). Marijuana/Cannabinoids: Neurobiology and Neurophysiology. CRC Press: Ann Arbor.

Crean JP, de Wit H, Richards JB (2000). Reward discounting as a measure of impulsive behavior in a psychiatric outpatient population. Exp Clin Psychopharmacol 2: 155-162.

Crean J, Richards JB, de Wit H (2002). Impulsive behavior in men with and without a family history of alcoholism: effects of tryptophan depletion. Behav Brain Res 136: 349-357.

de Wit H, Crean J, Richards JB (2000). Effects of D-amphetamine and ethanol on a measure of behavioral inhibition in humans. Behav Neurosci 114: 830-837.

de Wit H, Enggasser J, Richards JB (2002). Effects of D-amphetamine on behavioral measures of impulsivity in humans. Neuropsychopharmacology 27: 813-825.

de Wit H, Kirk J, Justice A (1998). Behavioral pharmacology of cannabinoids. In: Tarter et al (eds). Handbook of Substance Abuse: Neurobehavioral Pharmacology. Plenum Press: New York.

Derogatis L (1983). SCL-90-R Manual-II. Clinical Psychometric Research: Towson.
Diana M, Melis M, Gessa GL (1998). Increase in meso-prefrontal dopaminergic activity after stimulation of $\mathrm{CB} 1$ receptors by cannabinoids. Eur J Neurosci 10: 2825-2832.

Evenden JL, Ryan CN (1999). The pharmacology of impulsive behaviour in rats VI: the effects of ethanol and selective serotonergic drugs on response choice with varying delays of reinforcement. Psychopharmacology (Berl) 146: 413-421.

Evenden JL (1999). Varieties of impulsivity. Psychopharmacology 146: 348-361.

Green L, Fry AF, Myerson J (1994). Discounting of delayed rewards: a life-span comparison. Psychol Sci 5: 33-36.

Haertzen CH, Hickey JE (1987). Addiction research center inventory (ARCI): measurement of euphoria and other drug effects. In: Bozarth MA (ed). Methods of Assessing the Reinforcing Properties of Abused Drugs. Springer-Verlag: New York.

Hart CL, van Gorp W, Haney M, Foltin RW, Fischman MW (2001). Effects of acute smoked marijuana on complex cognitive performance. Neuropsychopharmacology 25: 757-765.

Johnson MW, Bickel WK (2002). Within-subject comparison of real and hypothetical money rewards in delay discounting. $J$ Exp Anal Behav 77: 129-146.

Johanson CE, Uhlenhuth EH (1980). Drug preference and mood in humans: diazepam. Psychopharmacology (Berl) 71: 269-273.

Kirk JM, de Wit H (1999). Responses to oral delta-9-tetrahydrocannabinol in frequent and infrequent marijuana users. Pharmacol, Biochem Behav 63: 137-142.

Logan GD, Sachar RJ, Tannock R (1997). Impulsivity and inhibitory control. Psychol Sci 8: 60-64.

Madden GJ, Petry NM, Badger GJ, Bickel W (1997). Impulsive and self-control choices in opioid-dependent patients and non-drugusing control participants: drug and monetary rewards. Exp Clin Psychopharmacol 5: 256-262.

Martin W, Sloan J, Sapira J, Jasinski D (1971). Physiologic, subjective, and behavioral effects of amphetamine, methamphetamine, ephedrine, phenmetrazine, and methylphenindate in man. Clin Pharmacol Ther 12: 245-258.

McNair D, Lorr M, Droppleman L (1971). Profile of Mood States. Educational and Industrial Testing Service: San Diego.

Meck WH (1996). Neuropharmacology of timing and time perception. Brain Res Cogn Brain Res 3: 227-242.

Mischel W, Shoda Y, Rodriguez ML (1989). Delay of gratification in children. Science 244: 933-938.

Mitchell S (1999). Measures of impulsivity in cigarette smokers and non-smokers. Psychopharmacology 146: 455-464.

Newman JP, Widom CS, Nathan S (1985). Passive avoidance in syndromes of disinhibition: Psychopathology and extraversion. $J$ Personality Soc Psychol 48: 1316-1327.

Patton JH, Stanford MS, Barratt ES (1995). Factor structure of the Barratt Impulsiveness Scale. J Clin Psychol 51: 768-774.

Richards JB, Sabol KE, de Wit H (1999a). Effects of methamphetamine on the adjusting amount procedure, a model of impulsive behavior in rats. Psychopharmacology (Berl) 146: 432-439.

Richards JB, Zhuang L, Mitchell SH, de Wit H (1999b). Delay or probability discounting in a model of impulsive behavior: effect of alcohol. J Exp Anal Behav 71: 121-143.

Roger RD, Owen AM, Middleton HC, Williams EJ, Pickard JD, Sahakian BJ et al (1999). Choosing between small, likely rewards and large, unlikely rewards activates inferior and orbital prefrontal cortex. J Neurosci 20: 9029-9038.

Rubia K, Russell T, Overmeyer S, Brammer MJ, Bullmore ET, Sharma $\mathrm{T}$ et al (2001). Mapping motor inhibition: conjunctive brain activations across different versions of go/no-go and stop tasks. Neuroimage 13: 250-261.

Schulze GE, McMillan DE, Bailey JR, Scallet A, Ali SF, Slikker W (1988). Acute effects of delta-9-tetrahydrocannabinol in rhesus monkeys measured by performance in a battery of complex operant tests. J Pharmacol Exp Ther 14: 178-186. 
Selzer ML (1971). The Michigan Alcoholism Screening Test: the quest for a new diagnostic instrument. Am J Psychiatry 127: $1653-1658$.

Solanto MV (1998). Neuropsychopharmacological mechanisms of stimulant drug action in attention-deficit hyperactivity disorder: a review and integration. Behav Brain Res 94: 127-152.

Sonuga-Barke EJ (2002). Psychological heterogeneity in AD/ HD - a dual pathway model of behavior and cognition. Behav Brain Res 130: 26-29.
Wade TR, de Wit H, Richards JB (2000). Effects of dopaminergic drugs on delayed reward as a measure of impulsive behavior in rats. Psychopharmacology 150: 90-101.

Wechsler D (1958). The Measure and Appraisal of Adult Intelligence. Williams \& Wilkins: Baltimore, MD.

United States Department of Health and Human Services National Household Survey on Drug Abuse (2000). Substance abuse and mental health administration. Washington, DC. 